\title{
Frontometaphyseal dysplasia
}

INSERM

\section{Source}

INSERM. (1999). Orphanet: an online rare disease and orphan drug data base.

Frontometaphyseal dysplasia. ORPHA:1826

Frontometaphyseal dysplasia (FMD) belongs to the otopalatodigital syndrome spectrum disorder and is characterized by anomalous ossification and skeletal patterning of the axial and appendicular skeleton, facial dysmorphism and conductive and sensorineural hearing loss. 